\title{
Risk Assessment Approaches for Carcinogenic Food Contaminants
}

\author{
Zoë Gillespie*, Olga Pulido and Elizabeth Vavasour \\ Bureau of Chemical Safety, Food Directorate, Health products and Food Branch, Health Canada, Canada \\ ${ }^{*}$ Corresponding author E-mail: Zoe.Gillespie@hc-sc.gc.ca
}

Received 13 April 2011; final version received 8 July 2011

Abstract Health Canada has identified the need for a standardized department-wide approach for the risk assessment of carcinogens in foods (e.g., pesticides, food chemical contaminants, veterinary therapeutics). A standardized approach would better facilitate and inform risk management strategies for the control of human exposure to food sources of carcinogens. Within the postmarket regulatory context, directly DNA-reactive carcinogens are of most concern because any exposure is theoretically assumed to be associated with a risk of producing a carcinogenic effect in proportion to the dose. Such non-threshold carcinogens, as well as carcinogens in which a non-linear dose response has not been demonstrated, require different approaches for risk characterization. In order to contribute to Health Canada's department-wide discussions regarding the development of risk management strategies for carcinogens, a general overview was conducted on international approaches for post-market risk assessments of carcinogenic contaminants in food. In this review, areas in the risk assessment paradigm which are identified for development of further standardized guidance include the weight-of-evidence determination for whether a compound should be considered a non-threshold carcinogen, the technical criteria for choosing the appropriate dose-response assessment approach, and a consistent approach for interpreting and prioritizing risk.

Keywords Carcinogenic, DNA-reactive, Non-threshold, Risk Assessment, Risk Management

\section{Introduction}

Health Canada projects have been initiated to establish standardized approaches for risk management of carcinogens. Healthy Environments and Consumer Safety Branch (HECS) through the Chemicals Management Plan (CMP) has been tasked with an initiative to establish an approach for the risk assessment and risk management of carcinogens under the Canadian Environmental Protection Act (CEPA). Since many substances being assessed under CEPA have uses that fall under statutes other than CEPA, including the Food and Drugs Act and the Pest Control Products Act, CMP is seeking wider consultation.

To assist them in developing their own policy regarding carcinogens, the Veterinary Drugs Directorate (VDD) of Health Canada led an earlier project to investigate how the risk assessment and risk management of carcinogens were conducted in HECS, the Pest Management Regulatory Agency (PMRA) and the Food Directorate (FD). A draft guidance document, entitled "Risk Analysis Framework" (2005), was produced to assist VDD evaluators in conducting cancer risk assessments in the pre-market context using a decision tree approach.

The pre-market context in which veterinary drugs are evaluated is similar to that of food additive evaluations by the Bureau of Chemical Safety (BCS) of the Food Directorate, in that risk management measures are not 
required for compounds that are both genotoxic and carcinogenic because such compounds are eliminated from consideration during the pre-market assessment. However, BCS is also responsible for the risk management of food contaminants (e.g. natural toxicants and food processing contaminants) that are evaluated in a post-market context. Consequently, approaches for the risk assessment of compounds that are both genotoxic and carcinogenic which were not considered in the VDD decision document need to be further elaborated to include the post-market risk management options.

Directly DNA-reactive carcinogens are considered to be of most concern because it is assumed that these substances do not demonstrate a dose below which they are not carcinogenic. More specifically, any exposure is theoretically assumed to be associated with some risk of producing a carcinogenic effect in proportion to the dose. Compounds that induce cancer by non-threshold modes of action or in which the threshold has not been clearly demonstrated require the use of different approaches in risk characterization.

Technological advances have made it possible to detect extremely low levels of contaminants in food. Consequently, risk perception and tolerance must also evolve because zero risk is not a realistic target for the post-market management of genotoxic carcinogens. Current risk assessment and management practices for genotoxic carcinogens are protectively conservative; however, these practices do not effectively incorporate the concept of prioritizing higher risk scenarios for management action. The need to prioritize contaminants for risk management action is an emerging issue required to ensure the most effective use of resources as the detection of contaminants in foods continue to improve.

This document is intended to assist Health Canada in establishing a department-wide approach to the risk assessment of genotoxic carcinogens in foods and develop risk management options by providing a discussion of internationally recognized approaches for the post-market risk assessment of food contaminants that are both genotoxic and carcinogenic.

\section{An Overview of the Risk Assessment Approach}

The evaluation strategy for the risk assessment process of adverse health effects resulting from human exposure to toxic substances in food is based on an internationally recognized risk assessment paradigm (NRC, 1983; WHO and FAO, 2009). Health Canada, including the Food Directorate, has used a comparable model for the risk assessment and risk management of mycotoxins in food (Health Canada Decision-Making Framework, 2000; Kuiper-Goodman, 2004).
Differences in the application of this risk assessment paradigm can be due to differences in one or more of the following: product type, context of risk (e.g. voluntary/involuntary exposure, extent of exposure, preexisting risk), risk tolerance, benefit assessment, support for international consistency within program areas, and availability of pre-market versus post-market information.

Recently, the U.S. National Research Council (NRC) has emphasized the design of risk assessments as a significant component of the risk assessment paradigm. The design of a risk assessment is defined as the process of planning a risk assessment and ensuring that its level and complexity are consistent with the needs to inform decision-making. In the early stages of a risk assessment planning and scoping and problem formulation are considered imperative. Planning and scoping involves discussion among decision-makers, assessors and stakeholders to establish issues to be assessed and the goals, breadth, depth and focus of an assessment. Problem formulation involves the development of a conceptual model and analysis plan for the assessment, and proceeds in parallel with planning and scoping (NRC, 2009; Abt et al., 2010). As noted by the U.S. Environmental Protection Agency (EPA), the incorporation of risk assessment design in the formative stages of an assessment may occur inconsistently and a formalization of this process is recommended to ensure the utility of risk assessments for decision-makers (Abt et al., 2010). Although, risk assessments conducted by Health Canada inherently include a similar process of contextualizing the safety and management issues, the formative design of risk assessments is not currently a formalized process.

For chemical agents, the risk assessment can be divided into:

Hazard identification - the determination of potential adverse health effects as a result of exposure to a substance. This determination is based upon a review of the toxicity data, which includes the results of toxicity testing in experimental animals, and any knowledge of effects on human health and mechanism/mode of carcinogenesis.

Hazard characterization - the determination of the doseresponse relationship and relevance to humans, incorporating factors such as interspecies variation in susceptibility and the relevance of mode/mechanism of action to humans.

Exposure assessment - the determination of the amount of human exposure to a substance. This determination uses data collected on the contaminant levels in food as well as specific food intake (consumption) information to calculate probable human exposure. 
Risk characterization - the estimation of risk to human health that is posed by exposure to a hazardous chemical. This procedure can be used to draw conclusions, regarding for example, what level of exposure to the hazardous chemical is associated with an increase in carcinogenic risk even if very small. In addition the risk characterization is used to inform risk managers what level of the risk may be acceptable or tolerable. To arrive at these estimates, consideration is given to the toxicity profile of the chemical in question, mechanisms of action, relevance to humans, dose-response and potential human exposure.

\subsection{Hazard Identification:}

In this context, the purpose of hazard identification is to evaluate the body of evidence from experimental and epidemiological studies and to identify whether or not the compound has: (a) carcinogenic potential and (b) genotoxic potential. Because carcinogenesis is a multistep process that can be affected by chemicals in a variety of ways, for simplicity it is assumed that the carcinogenic processes can be categorised into two major modes of action. The first is a direct DNA-reactive mode of action whereby the compound or its active metabolite(s) reacts covalently with DNA in target cells to form chemicalspecific addition products (adducts) which lead to procarcinogenic mutations, followed by neoplastic transformation and neoplasm induction. In addition, consideration should be given to whether or not a clastogenic compound induces chromosomal aberrations through a mutation event.

DNA-reactive carcinogens can be organized according to structural features, such as: alkenes, aromatic amines, nitrosamines, and polycyclic aromatic hydrocarbons (Williams, 2008)

The second mode of action is epigenetic in nature, in which the compound produces effects in target cells that either indirectly lead to neoplastic transformation or facilitate the development of neoplasms from cytogenetically transformed cells. The decision on whether or not the compound mediates genotoxic effects via direct DNA reactivity is considered the most important criterion in determining the nature of the dose response curve, i.e., linear versus non-linear. to hazard characterization (O’Brien et al., 2006).

Epigenetic carcinogens, which have diverse structures, can be organized according to mode of action, such as: promoters, endocrine modifiers, immunomodulators, cytotoxins and peroxisome proliferator-activated receptor agonists (Williams, 2008)

\subsubsection{Is the compound a carcinogen? \\ - A weight of evidence approach}

Determining whether a compound has carcinogenic potential is often based on neoplasia/tumour findings in a standard two-year carcinogenicity bioassay in rodents. Generally, well-designed two-year bioassays for carcinogenicity include adequate test doses in a sufficient number of animals and histopathological analysis of the appropriate organs in two rodent species. Key references to assess the adequacy of the study protocol are the OECD's monographs entitled "Guidance notes for analysis and evaluation of chronic toxicity and carcinogenicity studies" and "Draft guidance document No. 116 on the design and conduct of chronic toxicity and carcinogenicity studies, supporting TG 451, 452 and 453 (OECD, 2002; OECD 2009, GD No. 116). The latter reference highlights the broadened range and complexity of scientific data used to evaluate chemical toxicity and carcinogenicity potential for humans and in light of scientific progress the recently revised OECD test guidelines for carcinogenicity studies (TG 451), chronic toxicity studies (TG 452) and combined chronic toxicity/carcinogenicity studies (TG 453).

The determination of whether a compound has carcinogenic potential can be a complex issue. For example, the selection of the highest dose level to be used in a chronic toxicity or carcinogenicity study has long been a critical and sometimes controversial issue. The maximum tolerated dose (MTD), defined as the highest dose to produce toxic effects without causing death and to decrease body weight gain by no more than $10 \%$ relative to controls (OECD, 2002) is often used in the assessment of a chronic toxicity or a carcinogenicity study to decide whether the top dose tested was adequate to give confidence in a negative result. The high dose would be considered inadequate if neither toxicity or decreases in weight gain are observed. Failure to use an adequately high dose reduces the sensitivity of the studies (EPA, 2005a). In contrast, there are a number of concerns with the MTD, producing spurious effects that are not relevant to exposures expected to occur in humans (Table 1) (Dybing et al., 2002; US FDA, 2000). Many carcinogenicity studies can be challenged on the basis of selection of a top dose. This results in data that are difficult to interpret and may not be useful for regulatory purposes. 


\begin{tabular}{|l|l|}
\hline \multirow{5}{*}{ Physiological Effects } & - Saturating xenobiotic metabolising enzymes \\
\cline { 2 - 2 } & - Blocking detoxification pathways \\
\cline { 2 - 2 } & - Utilizing alternative pathway of metabolism \\
\cline { 2 - 2 } & - Interfering with hormonal balance \\
\cline { 2 - 2 } & - Altering of normal organ or cellular function \\
\hline \multirow{3}{*}{ Toxicokinetic Effects } & - Active transport mechanisms become saturated \\
\cline { 2 - 3 } & - Saturation of binding of chemical or active metabolites to plasma or tissue proteins \\
\cline { 2 - 3 } & - Decrease in cardiac output which decreases rate of tissue distribution and clearance \\
\hline \multirow{2}{*}{ Biochemical Effects } & - Inhibition or induction of enzymes \\
\cline { 2 - 3 } & - Depletion of cofactors \\
\hline \multirow{2}{*}{ Nutritional Effects } & - Interference with digestion or absorption of normal nutrients \\
\hline
\end{tabular}

Based on Dybing et al., 2002

Table 1. Potential Issues with the Use of MTD

For example, National Toxicology Program (NTP) 2-year gavage bioassays indicate that methyl eugenol, a compound that occurs naturally in a variety of spices, herbs and essential oils, induces hepatocellular carcinomas along with tumours at several other sites in rats and hepatocellular carcinomas and adenomas in mice. However, the relevance of these results remain in question because gavage administration of high doses delivered as a bolus coupled with rapid absorption (enhanced by gastric damage) represents an acute highlevel exposure of the liver, the main target organ. For many substances it has been shown that dosing by gavage can produce metabolic and toxicological effects that do not occur when the same daily dose is given in the diet (Smith et al. 2010).

Rhomberg et al., 2007 provides detailed guidance on criteria that can be applied in order to assess the acceptability of the high dose level or MTD. In addition, the U.S. EPA's cancer guidelines (2005a) provide general guidance for the interpretation of the significance and relevance of tumorigenic effects associated with dose levels below, at or above an adequate high dose.

An additional example of the complexity of the determination of whether a compound has carcinogenic potential for humans is the number of criteria considered when assessing the significance of the pathological results. These criteria include, evidence of carcinogenic effect in two different species, in both or just one sex, genotoxicity, common versus uncommon neoplasia, progression from adenomas to carcinomas, latency in tumour induction, multiplicity in site-specific neoplasia, occurrence of metastases, concurrent control and historical control incidence and statistical significance of tumour response. Issues may arise from the use of highly inbred rodent strains, with a high background incidence of strain-specific tumours (e.g. liver tumours in $\mathrm{C} 3 \mathrm{H}$ mice) or tumours arising from mechanisms not present in humans (e.g. $\alpha 2 \mathrm{u}$-globulin mediated kidney tumours in male rats). Assessment of the strength of the experimental evidence from two-year bioassays takes all of these criteria under careful consideration.

Although the decision of whether a compound has carcinogenic potential is often based on the standard twoyear carcinogenicity bioassay in rodents, other data can provide the basis for, or contribute to, the overall weight of evidence. For example, the compound may have been tested in alternative mouse models (e.g. p53+/-, Xpa -/-, rasH2 or $\operatorname{TgAC}$ ) or there may be evidence that the compound induces pre-neoplastic lesions, such as altered hepatic foci where the relationship between the lesion and eventual tumour formation is understood. In addition, epidemiological data may be available for chemicals with a history of exposure via industrial or other uses. When epidemiological data are available they are weighed preferentially to animal data in the weightof-evidence for determining carcinogenic risk for humans; however, good quality epidemiological data are rarely available.

In order to standardize the evaluation of the weight-ofevidence used to determine whether a compound has carcinogenic potential for humans, the International Agency for Research on Cancer (IARC) uses a categorization scheme for carcinogens based on the overall animal and human evidence. These categories 
evaluate the strength of evidence of carcinogenic risks to humans, and animals, and encompass the carcinogenic risks to experimental animals and humans, as well as mechanistic and other data. The descriptions of each classification scheme for humans and animals are not inclusive of all potential situations; further information is provided in the preamble to the IARC monographs on the evaluation of carcinogenic risks to humans (e.g. IARC monograph 97, 2008).

For carcinogenicity in experimental animals, IARC classifies the evidence of carcinogenicity as 'limited' when the data suggest a carcinogenic effect, but are limited for making a definitive evaluation such as: only one experiment was conducted; there were inadequacies in the design or conduct of the study, confounding the interpretation; or the incidence of only benign neoplasms or predominantly spontaneous tumours in susceptible strains was increased. With only poor quality studies available, a rating of 'inadequate evidence' is given. Good quality studies in two rodent species showing negative findings results in a rating of 'evidence suggesting lack of carcinogenicity'. Similarly, IARC rates evidence for human carcinogenicity based on epidemiological studies as 'sufficient' when causality has been clearly established, 'limited' when chance, bias and confounding factors cannot be ruled out, 'inadequate' for poor studies and 'evidence suggesting lack of carcinogenicity' for good studies with negative results. The IARC combines the ratings for the animal and human data giving an overall rating of group 1 ('carcinogenic to humans' - sufficient animal and human evidence); group 2A ('probably carcinogenic to humans' - limited human evidence and sufficient animal evidence, or inadequate human evidence and sufficient animal evidence with additional strong evidence that the carcinogenesis is mediated by a mechanism that also operates in humans or solely based on limited human evidence); group 2B ('possibly carcinogenic to humans' - limited human evidence and less than sufficient animal evidence, or inadequate human evidence and sufficient animal evidence, or inadequate human evidence and limited animal evidence with supporting evidence from other relevant data); group 3 ('not classifiable as to its carcinogenicity to humans' - inadequate human evidence and inadequate, or limited animal evidence, or sufficient animal evidence with strong evidence that the mechanism of carcinogenicity in animals does not operate in humans); and group 4 ('probably not carcinogenic to humans'). While the overall IARC rating system considers the genotoxicity and possible mode(s) of action of a chemical, the rating system does not explicitly state whether the weight of evidence considers the chemical to have a threshold or a non-threshold carcinogenic mode of action. The IARC rating system focuses on hazard identification rather than risk characterization.
Under the Canadian Environmental Protection Act (CEPA, 1994), Health Canada has developed a carcinogen-ranking scheme based on the IARC ranking scheme. CEPA's scheme consists of more categories and subcategories and is not totally consistent with those of IARC and USEPA. CEPA distinguishes between genotoxic and non-genotoxic carcinogens, and gives the latter group a lower ranking when epidemiological evidence is inadequate. The criteria for classification of carcinogenicity (Appendix B) and mutagenicity in germ cells (Appendix C) is available on the Internet at: http://www.hc-sc.gc.ca/ewh-semt/pubs/contaminants/ approach/index-eng.php.

Once all available data are collated and considered as a whole, a weight-of-evidence decision can be made on whether a compound has carcinogenic potential for humans. Food contaminant evaluations conducted by BCS frequently cite, when available, the IARC classification of a compound as part of the overall hazard identification. It is recommended that a department-wide standard to categorize the available weight-of-evidence to determine whether a compound has carcinogenic potential for humans, is adopted or established similar to that used by IARC, to increase transparency and consistency for weight -of-evidence based decisions.

\subsubsection{Is the carcinogen genotoxic? \\ - Directly DNA-reactive vs. epigenetic}

The approach adopted in the risk assessment to identify a compound as directly DNA-reactive or epigenetic can be a complex issue. The overall weight-of-evidence is based on information from various studies, none of which is sufficient by itself.

According to generally accepted guidelines ${ }^{1}$, the standard three-test battery for the detection of genotoxic compounds consists of: (i) an in vitro test for gene mutation in bacteria with and without metabolic activation; (ii) an in vitro test in mammalian cells with cytogenetic evaluation of chromosomal damage (and/or a test that detects gene mutations); (iii) an in vivo test for chromosomal damage in rodent hematopoietic tissue. This test battery is designed to identify the majority of genotoxic substances.The selection of the assays to be included in the standard test battery took into consideration various criteria including, but not limited to, the reliability and validity of the assays, the genetic

\footnotetext{
${ }^{1}$ For example: International Conference on Harmonization of Technical Requirements for Registration of Pharmaceuticals for Human use (ICH Guidelines); US Food and Drug Administration Center for Food Safety and Applied Nutrition Office of Premarket Approval (Redbook); Canadian Environmental Protection Act, 1999; Organization for Economic and Cooperative Development (OECD Guidelines); US EPA guidelines for toxic substances and pesticides
} 
characterization or types of genetic damage detected, the sensitivity of the assays to different subsets of chemicals, the ability of the assay to detect a broad range of genetic events and provide maximum information. Use of the standard test battery generates a body of data on which to base decisions about either the need for further testing and/or the degree of concern about the potential mutagenicity of the test agent (Dearfield et al., 1991)

Some limitations to these tests have been identified which may result in a failure to identify some genotoxic substances. The failure of the standard test battery in detecting genotoxic activity of some carcinogens is attributable to several causes, in particular: the limited spectrum of metabolic activity of the liver S9-mix used in in vitro studies, and the differences in biotransformation of chemicals in cells of different type and cells from different animal species; in vivo, the pharmacokinetic behaviour of the test compound and its possible species-, sex- and tissue-specificity can affect whether the target cells are adequately exposed for a sufficient period of time (Brambilla and Martelli 2004).

It is also important to emphasize that data from genotoxicity studies may show that a compound is genotoxic but not directly DNA-reactive. For example, epigenetic effects such as the induction of numerical chromosome aberrations via interference with the microtubules at cell division can either indirectly result in DNA modification leading to procarcinogenic mutations or facilitate development of preexisting neoplastic cells into neoplasms. Epigenetic substances produce their carcinogenic effects via mechanisms that would be expected to show a non-linear dose response (Williams, 2008; O'Brien et al., 2006).

$$
\begin{aligned}
& \text { Examples of } 4 \text { Epigenetic Carcinogens: } \\
& \text { butylated hydroxyanisole } \\
& \text { d-limonene } \\
& \text { phenobarbital } \\
& \text { 2,3,7,8 - tetrachlorodibenzo-p-dioxin }
\end{aligned}
$$

Other assay systems can provide information on how a compound interacts with DNA. These studies measure direct interaction with DNA (e.g. DNA binding studies and DNA adduct formation) and indirect measures of DNA damage (e.g. the alkaline elution, single cell gel electrophoresis (comet) and DNA repair assays, in the absence of general cytotoxicity) (Brusick, 2001; O'Brien et al., 2006). Examining whether the compound is structurally related to other known directly DNA-reactive genotoxic carcinogens may also provide insight when determining whether a compound is a direct DNAreactive genotoxicant.

$$
\begin{aligned}
& \text { Examples of } 3 \text { Chemical Classes of } \\
& \text { DNA-reactive carcinogens: } \\
& \text { polycyclic aromatic hydrocarbons } \\
& \text { nitrosamines } \\
& \text { heterocyclic amines }
\end{aligned}
$$

In addition to genotoxicity data which is useful for the identification of these compounds, information on the metabolic fate and mode of action of a compound is considered pivotal information when attempting to decipher whether a compound is directly DNA-reactive or epigenetic.

Based on available data for specific chemicals, the EPA and the International Programme on Chemical Safety (IPCS), have developed approaches aimed at understanding the mode of action underlying induced tumorigenesis in experimental animals, and whether this mode of action is relevant to humans (EPA, 2005; SonichMullen et al., 2001). This approach has been expanded into the Human Relevance Framework (HRF), which also uses a weight of evidence approach to hypothesize a mode of action (Meek et al., 2003; Meek, 2008). The mode of action is used to describe 'key events' and processes underlying toxicity including carcinogenicity, whereas 'mechanism of action' implies a more detailed molecular description of events. Key events are defined as necessary events that are on the causal pathway toward cancer and are distinguished from 'associated events' that may occur at the same time. Species and animal strain susceptibility play an important role in the mode of action (EPA, 2005; Schlosser and Bogdanffy, 1999; Dybing et al., 2002).

Under the HRF approach, as well as under EPA guidelines, it is presumed that cancer induced in animals by both DNA-reactive and epigenetic compounds is relevant to humans by default, unless the mode of action indicates otherwise. Unfortunately, mode of action information is not always available. Key references on the critical assessment of mode of action of a chemical carcinogen are entitled: "IPCS Conceptual framework for evaluating a mode of action for chemical carcinogenesis."; “Overview: Using mode of action and life stage information to evaluate the human relevance of animal toxicity data."; "Guidelines for Carcinogen Risk Assessment"; and "IPCS framework for analyzing the relevance of a cancer mode of action for humans."; (Sonich-Mullin et al., 2001; Seed et al., 2005; EPA, 2005a; Boobis et al., 2006) 
Once all available data are collated and considered as a whole, a weight-of-evidence decision can be made on whether a compound is directly DNA-reactive or epigenetic. In cases where the weight-of-evidence for genotoxicity is inconclusive and a substance has been shown to be carcinogenic but its carcinogenic mode of action has not been identified, or the mode of action has been identified but there is insufficient supporting evidence, the default assumption is that the mode of action leading to cancer has a linear dose response.

In the BCS health risk assessment of ochratoxin A (OTA), a natural toxin produced by fungi, OTA is recommended to be regulated as a non-threshold carcinogen despite ongoing international debate about the genotoxic status of OTA and the undetermined mode of action (KuiperGoodman et al., 2010).

It is recommended that departmental-wide standards are developed to increase consistency in the weight of evidence required to differentiate between directly DNAreactive or epigenetic compounds and to define the weight of evidence required to determine and substantiate whether a mode of action is directly DNAreactive or epigenetic.

\subsubsection{Does the mode of action have a threshold? \\ - True vs. Apparent}

The threshold determination for genotoxic carcinogenic compounds generally presumes that there is no threshold dose below which there is no induction of cancer initiation because the mode of action may involve a single direct reaction, specifically, a single hit in a single target (Kirsch-Volders et al., 2000). Thus there would always be some risk, even at very low doses, unless it can be clearly established that the mode of action involves an indirect mechanism that may have a threshold.

In contrast to the presumed dose-response of DNAreactive carcinogens for which no threshold can be defined, different types of thresholds for genotoxic carcinogens are defined in the literature (Table 2).

\begin{tabular}{|l|l|l|}
\hline Type of Threshold & Defined in the Literature & Reference \\
\hline perfect & based on non-genotoxic carcinogens & Hengstler et al., 2003 \\
\hline real & based on non-genotoxic carcinogens & Kirsch-Volders et al., 2000 \\
\hline statistical & based on mitotic spindle poisons & Kirsch-Volders et al., 2000 \\
\hline apparent & $\begin{array}{l}\text { based on rapid degradation (toxicokinetics) of the chemical or to } \\
\text { other/additional factors that limit target exposures }\end{array}$ & Kirsch-Volders et al., 2000 \\
\hline practical & $\begin{array}{l}\text { based on idea that chemical should cause no genotoxic effect at } \\
\text { very low or immeasurable target concentrations }\end{array}$ & $\begin{array}{l}\text { Hengstler et al., } 2003 \text { and } \\
\text { Seiler, 1977 }\end{array}$ \\
\hline
\end{tabular}

Based on Bolt and Degen. (2004)

Table 2. Types of thresholds for carcinogens are defined in the literature

\begin{tabular}{|ll|}
\hline • & Incomplete absorption/rapid excretion \\
\hline • & Binding to extracellular molecules \\
\hline • & Dilution upon systemic distribution among $6 \times 10^{13}$ cells in body \\
\hline - & Low probability of reaching target stem cell \\
\hline - & Limited cellular uptake/efficient elimination at target site \\
\hline - & Reaction with non-DNA nucleophiles \\
\hline - & Reaction with non-utilized regions of DNA, among the $3 \times 10^{9}$ base pairs per cell \\
\hline - & Efficient DNA repair prior to replication \\
\hline - & Low probability of producing transforming mutations in multiple critical genes \\
\hline$\bullet$ & Infrequency of neoplastic development from preneoplastic lesions \\
\hline
\end{tabular}

Based on Williams et al. (2005)

Table 3. Factors that limit the carcinogenicity of DNA-reactive carcinogens 
In order to simplify terms, Bolt and Degen (2004) proposed that true thresholds include perfect thresholds (as defined by Hengstler et al., 2003) and both real and statistical thresholds (as defined by Kirsch-Volders et al., 2000) all of which correspond with nongenotoxic/epigenetic modes of action. Definitions of apparent (Kirsch-Volders et al., 2000) or practical thresholds (Hengstler et al., 2003; Seiler, 1977) are based on the concept that various factors (Table 3 ) can restrict the potential carcinogenicity of DNA-reactive carcinogens (Williams, 2008). When sufficient information is available to distinguish between these different types of thresholds (True Threshold, Apparent Threshold or No Threshold) for carcinogens, this determination can contribute to the characterization of the hazard to humans and help to decide the approach employed in the dose-response assessment. Currently, BCS does not use these terms in every risk assessment of genotoxic and carcinogenic compounds. The development of departmental-wide standards for the use of consistent definitions and terminology is recommended.

\section{Hazard Characterization (Dose-Response Assessment)}

Hazard characterization can be described as a process that involves the qualitative and/or quantitative evaluation of the nature of the adverse effects that humans may experience under expected levels of exposure to an agent (Health Canada, 2000). A range of different approaches may be needed depending on the data available and the hazard or risk characterization issue.

\subsection{Considerations when Deriving Health-based Guidance Values}

The goal of hazard characterization for compounds for which a threshold or an apparent threshold (supported by studies on mechanism and/or toxicokinetics) is substantiated is the estimation of a 'safe dose', such as a tolerable daily intake (TDI), equivalent to the acceptable daily intake (ADI) and the chronic oral reference dose (RfD). The TDI is the dose that can be safely consumed daily over a lifetime without incurring appreciable adverse health effects, and which by implication therefore involves a biologically insignificant risk (WHO 1987, 1999, Edler et al., 2002, Kuiper-Goodman, 2004, EHC 240, 2009).

As an initial step, responses associated with carcinogenesis, as identified under hazard identification, are examined using biological criteria for relevance to humans. This is followed by an examination of the relationship between dose and observed responses in carcinogenic assays, and an extrapolation of dose from the animal species to humans. In the carcinogenic assays, the no-observed-adverse-effect-level (NOAEL) of the most sensitive but relevant adversely affected endpoint, called the critical effect, in the most sensitive species is selected. If adverse effects are observed in animals receiving the lowest dose, the lowest-observed-adverseeffect-level (LOAEL) is selected.

To derive a TDI, it has been common practice to divide the NOAEL/LOAEL by safety/uncertainty factors. The 'safety /uncertainty factor' takes the following into consideration: inter-species differences, inter-individual differences, quality/adequacy of the database, selection of LOAEL versus NOAEL, severity of toxicity and sensitivity/concern for specific populations. The IPCS has published a "Guidance document for the use of data in development of chemical-specific adjustment factors (CSAFs) for interspecies differences and human variability in dose/concentration response assessments" as efforts have been made to replace the inter-species and inter-individual (intraspecies) factors with chemical specific factors where toxicokinetics and toxicodynamic differences between species and individuals are addressed (WHO, 2005). This approach can be used when sufficient data are available to support the use of chemical-specific adjustment factors.

The NOAEL represents only one point on the observational portion of the dose response curve. Since the number of dose groups in a study, the spacing of dose groups and group size all influence the empirical derivation of the NOAEL, it has been argued that it may be more appropriate to derive mathematically the 'true' point of departure in the dose-response relationship (Edler et al., 2002). One such estimate is the derivation of the benchmark dose (BMD) and its lower confidence interval (BMDL).

In 2008, BCS used BMD modelling to identify a predetermined point of departure response for melamine, a chemical contaminant identified in infant formula, milk and milk ingredients. Melamine was determined to have a threshold mode of action. The point of departure was used to derive a toxicological reference value (TRV) used in the risk characterization of the theoretical melamine contamination and in setting interim maximum limits (MLs) which did not constitute a health risk for consumers $^{2}$. Canada, through work with the Codex Committee on Contaminants in Food (CCCF), helped develop standards for melamine in food and infant formula.

The decision to use BMD modelling is dependent on the data available and the specific hazard or risk characterization issue. BMD modelling is further discussed in the subsequent section "Considerations when employing mathematical modelling".

\footnotetext{
${ }^{2}$ http://www.hc-sc.gc.ca/fn-an/pubs/melamine_hra-ers-eng.php
} 
With the increasing quality and subsequent application of mode of action data to the risk assessment approach, support for a bioindicator-based risk assessment (BIBRA) approach has been discussed in scientific literature (Williams, 1999; 2001; 2008). This approach has not been applied in a food contaminant risk assessment.

The BIBRA focuses on selecting a critical bioindication of cellular (toxic) effect that is the basis for experimental carcinogenicity. For threshold carcinogens, it has been proposed that using the NOAEL for an essential cellular effect in the pathogenesis of epigenetic neoplasia provides an additional margin of safety (Williams, 2008). The selection of a reference point such as sustained hyperplasia of a type known to lead to neoplasia could be used to derive what may be regarded as 'toxicologically insignificant daily intake (TIDI)'. A further extension of the BIBRA would be to identify the gene transcriptional events related to the bioindicator of effect and use what has been referred to as a 'no observed transcriptional effect level (NOTEL) (Lobenhofer et al., 2004) as the NOAEL. However, this approach may be overly conservative as changes in transcriptional activity may not always be translated into differences in protein levels or protein activity. Conventional uncertainty/safety factors would be applied to the selected NOAEL to derive the TIDI. Consideration could also be given to reducing the default uncertainty/safety factors using the CSAFs approach noted previously.

Williams (2008) also suggests that the BIBRA approach could be applied to DNA-reactive carcinogens. If a NOAEL for chemical-specific DNA adducts is identified using appropriately sensitive experimental methods, this NOAEL could be used as the bioindicator to calculate a TIDI by applying appropriate uncertainty/safety factors. For DNA-reactive carcinogens, adduct levels have been demonstrated to be highly predictive of tumour initiation but occur at doses below those that elicit carcinogenicity and even in cases where carcinogenicity is not found (Sander et al., 2005). Williams (2008) suggests that a TIDI based on adduct data of less than 1 in $10^{9}$ nucleotides could be considered protective of potential carcinogenicity.

It is noted that the BIBRA approach requires additional work before it could be applied in a food contaminant risk assessment, as internationally validated test guidelines to measure levels of transcriptional changes or DNA adducts have not been established.

\subsection{Considerations when Employing Mathematical Modelling}

For directly DNA-reactive carcinogens or carcinogens in which the mode of action can not be identified or substantiated, the default position has been that there is a linear dose response for effects, such as initiation of the carcinogenic process. A TDI, which is based on a threshold such as the NOAEL, is not considered appropriate. Non-threshold carcinogens are not permitted for deliberate addition to food but when such compounds are detected in food and their presence cannot be completely prevented, a variety of approaches can be used to determine exposure levels that may be so low as to not be of concern.

Appropriate mathematical models, most of which presume a linear dose-response relationship at low doses, have been used to extrapolate from the observable part of the dose-response curve to low doses. These models do not take into account relevant data on mechanisms of tumour induction or differences in susceptibility/dynamics between the animal species and humans, or differences in susceptibility between individuals. However, there is international agreement that if there are sufficient data of good quality on a substance, then dose-response modelling should be carried out and that the selection of an appropriate reference or point of departure should be driven by the extent and quality of the available data (Barlow et al. 2006). This consensus was reached at an international conference organized by EFSA and WHO with support of ILSI Europe (EWI conference) held in 2005 on "the risk assessment of substances that are both genotoxic and carcinogenic". Representatives from various academic institutions, food industries and international regulatory agencies, including Health Canada, were in attendance. A summary report of the EWI conference proceedings was published in 2006 in the journal of Food and Chemical Toxicology (Barlow et al., 2006).

There was a consensus at the EWI conference that the benchmark dose (BMD) approach offers the best tool for deriving a reference point/point of departure within the observable dose range. The BMD approach can be applied when there are as few as two dose groups showing tumours and one control group, but it has been noted that a larger number of dose groups, at least 3 dose groups showing tumours plus a control, would be preferable (Edler et al., 2002). The BMD approach makes full use of all the data points on the dose response curve; a mathematical model is fitted to the experimental data within the observable dose range and the dose that causes a low but measurable response is chosen as the BMD. BMDs can be modelled for each tumour type and for overall tumour incidence. The BMD lower limit (BMDL) refers to the one-sided lower 95\% confidence limit of the BMD. If the data are sufficient to enable a BMDL to be determined with confidence, then this is the most appropriate reference point/point of departure because the BMDL better reflects the greater uncertainties of lesser amounts of data, since the $95 \%$ confidence interval on the BMD is wider when there are fewer data. A BMDL10 was considered preferable to a BMDL5 
because the BMDL10 is more likely to be within the observed dose range and as such does not require extrapolation. Data from any one substance has shown that the various BMDL10 values obtained from different dose-response models are in closer agreement than BMDL5 values. Although there is no general agreement, it has also been suggested that when there is a factor of more than 100 between the BMD and the BMDL values, this reflects considerable uncertainty and the BMDL should not be used as a reference point/point of departure (Barlow et el., 2006).

The BMD approach -

Is usually based on calculation of the BMDL10 - the $95 \%$ lower confidence interval on a benchmark dose (BMD) for a $10 \%$ increase in tumour incidence. Values for BMDL10 are determined by fitting doseresponse data to various mathematical models. (Dybing et al., 2008)

Carcinogenic potency estimates, such as the T25, can also be used as reference points/points of departure. Carcinogenic potency estimates give an indication of the dose of a substance administered over a standard animal lifespan that results in a fixed incidence of tumours, such as, 5,25 or $50 \%$, for the TD05, T25 and TD50 respectively, after correction for the spontaneous background incidence of tumours among controls. In common with the BMD approach, carcinogenic potency estimates also make use of all the available dose-response data. If the data are insufficient to derive a BMDL10, the EWI conference agreed that use of the T25 was an alternative option. The minimum data requirements to calculate a T25 are one incidence level significantly greater than the controls. The T25 method can be used to compare and rank substances for their carcinogenic potency, to calculate MOEs (discussed under risk characterization), or to estimate risks at lower doses by linear extrapolation (Table 4). It was noted that the T25 method has been used in risk assessment for regulation of non-food genotoxic and carcinogenic chemicals in the EU. The T25 can be calculated without computing the dose response curve whereas the TD50 method requires specific software. Also noted was that linear extrapolation from the TD50 can seriously over or underestimate the true risk and that it is more often used only for comparing carcinogenic potencies.

\section{The T25 approach-}

The T25 value is the chronic daily dose, which will give tumours in $25 \%$ of the animals above background at a specific tissue site. The T25 is determined by linear extrapolation from the lowest dose giving a statistically significant increase in tumours (the critical dose) (Dybing et al., 2008)
- for comparing the potency of different compounds

- to extrapolate the incidence down to the level of human exposure

- to estimate an intake that would give a very low risk

- to compare directly with the level of human exposure by calculation of the margin of exposure

Based on Dybing et al., 2008

Table 4. Uses for the BMDL10 and T25

For low dose linear extrapolations, a more complex mathematical model may be fitted to the data points to estimate the risk at lower doses. However, simple linear extrapolations can be carried out from either a selected incidence within the experimental range or from a derived incidence, such as one of the points of departure described above (BMDL10 or T25) obtained by fitting a mathematical model to the observed data. When using these approaches, low risk is often defined, for example, as the level of exposure to a substance associated with an upper bound, lifetime risk of cancer of 1 in a million persons $\left(1 \times 10^{-6}\right)$ (i.e. for pesticides and some food contaminants) or one in a hundred thousand persons $(1 \mathrm{x}$ $10^{-5}$ ) (i.e. mycotoxins). It is noted that the acceptance of a risk level is a risk management decision.

The EWI conference noted that different mathematical models, which provide equally good fits to the experimental animal data, can result in very different estimates of risk at low doses. The conference also noted that the mathematical models tend to be highly conservative and therefore linear extrapolation should be considered as giving an upper bound estimate rather than the most likely estimate of risk.

The BCS has used a range of different approaches in the dose-response assessment of genotoxic carcinogens because as previously stated, the approach used depends on the data available and the hazard or risk characterization issue. In 2005, BCS conducted a risk assessment for malachite green (MG), an antifungal agent not permitted for use in food-producing animals based on scientific evidence indicating that a metabolite, leucomalachite green (LMG), may be a non-threshold, genotoxic carcinogen. Due to industrial applications of MG in pulp and paper and textile manufacturing residues of MG were being detected at varying levels in both imported and domestic commercial fish. The induction of hepatocellular adenomas and carcinomas in female mice treated with LMG were considered to be the pivotal effect. BMD modelling of the liver tumour data was used to derive a BMDL10 as the point of departure on the dose 
response curve. In 2006, a BCS risk assessment for benzene was initiated due to trace levels forming in beverages under certain conditions when ascorbic acid combines with either sodium or potassium benzoate. Because there is insufficient evidence to substantiate a threshold mode of action, benzene is regulated as a nonthreshold genotoxic carcinogen. BMD modelling was not applied in the hazard characterization because the tumour incidence observed in animal studies was not consistent with the cancer endpoint in humans (i.e. acute non-lymphocytic leukaemia) and there was a lack of a dose response in the animal data. Consequently, the USEPA linear extrapolation method using a human cancer endpoint to derive an oral slope factor for benzene (i.e. cancer risk per unit dose) was used to calculate the oral exposure associated with a lifetime cancer risk level of 1 in a million ${ }^{3}$. In the 2009 BCS risk assessment for the mycotoxin, OTA, the TD05 was used as the point of departure on the dose-response curve (Kuiper-Goodman et al., 2010). The TD05 was divided by a factor of 5000 to derive a value considered equivalent to a response level of $1 \times 10^{-5}$ in animals. This approach provides a similar estimate of safe intake to that derived using low dose linearized models (Kuiper-Goodman, 2004).

In agreement with the EWI conference, the choice of which approach to follow should be case-by-case, based on consideration of the extent and quality of the available data. It is recommended that department-wide guidance be developed that outlines the technical criteria for choosing between the various approaches, including the advantages and disadvantages of the various approaches and the minimum data sets required for each approach.

\section{Exposure Assessment}

Intake scenarios for genotoxic carcinogens in food include naturally occurring substances, environmental contaminants, substances used illegally as food additives or veterinary drugs or substances formed during cooking or processing, since substances authorised for deliberate addition to foods, such as food additives, exclude genotoxic carcinogens. There are two distinct elements to the estimation of dietary exposures (a) analytical measurement of the compound in foods and (b) measurement of intakes of foods that may contain the chemical (food consumption data) (O’Brien et al., 2006).

\subsection{Measuring the Compound in Food}

The low levels at which genotoxic carcinogens are usually present in foods may present special challenges to the analytical technology used. It is important that validated standard methods be employed where possible. Detailed

3http://www.hc-sc.gc.ca/fn-an/securit/chem-chim/foodaliment/benzene/benzene_hra-ers-eng.php guidelines are available regarding the handling of data from food analyses for the purpose of risk assessment (WHO, 1995; Douglass and Tennant, 1997; Barlow et al., 2002a,b; Petersen, 2002; Renwick et al., 2003). WHO (1995) recommended that analytical efforts be targeted at foods contributing to the majority of exposure to a contaminant. Monitoring should concentrate on appropriate dietary staples that are likely to be the major sources of intake. Distinct geographic regions or ethnic food consumption patterns may necessitate different monitoring plans. Another factor to consider is that genotoxic carcinogens may not be evenly distributed in the food matrix; examples include, surface mould contamination (aflatoxin B1) or the charred surfaces of meat (benzo(a)pyrene). It is essential that foods analysed are representative of foods consumed by the population(s) or sub-populations of interest $\left(\mathrm{O}^{\prime}\right.$ Brien et al., 2006).

\subsection{Collecting Food Consumption Data}

Food consumption data can be obtained following three broad approaches: analysis of food supply data, household data and individual food consumption surveys. Country or region specific food consumption data are preferable but surrogate data can be used when such specific data are not available. For example, when Canada specific data are not available the data from the US Continuing Survey of Food Intake by Individuals (CSFII) would be considered relevant to Canadian consumption figures in many instances.

Food supply data measure the availability or disappearance of foods on a national or regional basis each year, which can be expressed as an estimate of mean per capita consumption. Although such data are crude, they may be the only form of data available for some countries and can facilitate comparison of dietary exposures for different countries and regions. The WHO GEMS/Food programme ${ }^{1}$ prepared a series of regional diets for conducting risk assessments based on food supply data. Household surveys may be regarded as an extension of national food supply surveys applied at household level. While much useful detail can be obtained from such data, food losses due to waste, spoilage, cooking and preparation are generally ignored, the amount of food consumed by individuals is not identified and foods consumed outside the home are not measured. Although the most accurate food consumption data are acquired in studies of individual food consumption, uncertainties still exist. Food frequency data allow only qualitative estimates of exposure and are of limited utility for the accurate estimate of chemical intakes. Food recall data depend on the memory of the respondent to relay the types and quantities of food

\footnotetext{
${ }^{1}$ www.who.int/foodsafety/chem/gems/en/index.html
} 
consumed. Longer-term food recalls allow for the frequency of consumption to be estimated, which is, along with portion size, essential information in the determination of the average daily intake of a given food. Food diaries such as seven day weighted intakes provide quantitative data on foods consumed but are labour intensive ( $\mathrm{O}^{\prime}$ Brien et al., 2006).

Whichever method is used to derive food consumption data, the risk characterization for genotoxic carcinogens typically involves the combination of lifetime animal potency data with data that are representative of the pattern of chronic exposure and exposure among subpopulations of toxicological interest. In cases when the exposure scenario is short-term, acute or intermittent the use of adjustment factors and/or averaging exposure over a lifetime have been proposed by various regulatory organizations including the EPA and Health Canada. According to the EPA supplemental guidance for assessing susceptibility from early-life exposure to carcinogens, people exposed to carcinogens with a mutagenic mode of action during early life are assumed to have increased susceptibility. The EPA guidance recommends the application of age-dependent adjustment factors in the absence of chemical-specific data (EPA, 2005b). Health Canada has recently conducted a literature review to evaluate whether averaging shortterm exposure over a lifetime would be adequate to estimate cancer risk using cancer slope factors derived from chronic animal studies (Li-Muller et al., 2011 draft). As an interim position, it was considered that the application of age-dependent adjustment factors, as proposed by the EPA (EPA, 2005a,b), to the cancer slope factor with exposure averaged over a lifetime to provide generally conservative estimates of lifetime cancer risks. As noted in the draft position paper, evidence from animal experiments, epidemiologic studies and theoretical modelling studies support that the averaging less-than-lifetime exposure over a lifetime (LADD) may underestimate or overestimate cancer risks, depending on the timing of exposure and the mode of action of the carcinogen. The degree of underestimation is reported to be within an order of magnitude (Li-Muller et al., 2011 draft). In a review of the derived cancer risk from shortterm, high-dose exposure to a genotoxic carcinogen relative to the same cumulative dose distributed over a lifetime (virtually safe dose), although data was limited, dose rate correction factors (factors by which a specified dose of a carcinogen at long term, low dose rates should be multiplied to derive the expected tumour incidence from short term, high dose rates) ranged from unity to 8.3 (Bos et al., 2004). Bos et al., (2004) concluded that the most pragmatic approach to calculate acceptable shortterm exposures to known genotoxic carcinogens is to linearly extrapolate the short-term exposure from the acceptable lifetime exposure or virtually safe dose. Health Canada continues to review work conducted in this area and has not developed a formalized policy for less-thanlifetime exposure scenarios at this time.

\section{Risk Characterization}

Risk characterization is the (a) qualitative and/or (b) quantitative estimation, including attendant uncertainties, of the probability of occurrence and severity of known or potential adverse health effects in a given population based on hazard identification, hazard characterization (dose-response assessment) and exposure assessment.

\subsection{Qualitative Approaches}

An approach that advises risk managers that the intake of genotoxic carcinogens should be 'As Low As Reasonably Achievable' (ALARA) requires only the weight-ofevidence identification of the compound as a genotoxic carcinogen. Although the ALARA principle is an easy concept to understand, it is generally agreed that it poses some major difficulties for the risk manager as it does not discriminate between very potent and very weak carcinogens and does not take human exposure into account. Improved analytical techniques with lower detection limits, extended testing and increased surveillance have allowed the identification of substances in food at very low levels. As such, ALARA does not provide an estimate of risk and does not give risk managers sufficient information to assess the degree of urgency and extent of risk reduction measures that may be required. Furthermore, ALARA does not allow risk managers to conduct comparisons between different compounds in order to aid in the establishment of priorities for risk management action.

In cases where the exposure to a food contaminant is unavoidable, for example a substance is present at background levels in the environment, the Food Directorate takes the position that levels of contaminants should be minimized as much as possible according to the ALARA approach. However, the ALARA approach is not used for risk characterization but rather as a principle to guide risk management options. BCS typically employs quantitative approaches discussed in the next section.

\subsection{Quantitative Approaches}

For compounds with data that substantiate a threshold, risk characterization involves a comparison of levels of daily exposure over a lifetime to the TDI, the intake for which the risk is considered to be insignificant. If longterm or short-term exposure exceeds the TDI, recommendations for risk reduction may be made, with priorities determined by the extent to which the TDI is exceeded (Kuiper-Goodman, 2004). 
For non-threshold compounds or compounds without sufficient acceptable evidence to support a threshold mode of action, the numerical estimate of risk associated with human exposure can be derived by extrapolation of the animal dose-response data or by the use of the TD50, T25 or BMDL10 as the point of departure for a low-dose risk estimation by simple linear extrapolation (discussed under Hazard Characterization). A major problem with the generation of theoretical numerical risk estimates is that they may be misinterpreted as a realistic indication of actual risk rather than an upper bound estimate.

The threshold of toxicological concern (TTC) approach is advocated in scientific literature for risk assessment of contaminants in food in cases where the biological data are few but the chemical structure is known and there are good exposure data (e.g. chemicals migrating from food packaging materials for which data indicate it is directly DNA-reactive but for which there are no data from a cancer bioassay or the carcinogenicity data fail to define a dose-response relationship) (Kroes et al., 2002; O'Brien et al., 2006). In this approach, if the substance is directly DNA-reactive or has a structural alert for DNA-reactivity and does not belong to a group of identified structures that are likely to be the most potent directly DNAreactive carcinogens, exposures below 0.15 $\mu \mathrm{g} /$ person/day (or $0.0022 \mu \mathrm{g} / \mathrm{kg} \mathrm{bw} /$ day assuming an average body weight of $70 \mathrm{~kg}$ ) are considered to be of negligible risk. This figure is based on the daily intake of a compound estimated to pose a lifetime risk for the development of cancer of less than one in a million, using the highly conservative approach of linear extrapolation from the TD50 values derived from the dose-response data from rodent cancer bioassays on all available structurally related compounds studied in rodent cancer bioassays.

Although the TTC approach is not currently used to regulate directly DNA-reactive substances, the European Food Safety Authority's Panel dealing with food contact materials applies a tiered approach to safety testing requirements that has some similarities with the philosophy of the TTC approach. For example, in the case of substances for which based on migration data the content in food is assumed not to exceed $50 \mathrm{ppb}$ (equivalent to $150 \mu \mathrm{g} /$ person/day) 3 in vitro genotoxicity tests are required. If these assays are negative, it is assumed that there will not be adverse effects at the highest dietary exposures that may be encountered (EC, 2008).

In a post-market context, BCS has not used the TTC approach. However a TTC-like approach, modelled after the US FDA “Threshold of Regulation" policy is used for pre-market food packaging assessments. An estimated value of $0.5 \mathrm{ppb}$ (equivalent to $1.5 \mu \mathrm{g} /$ person/day) in food was derived and implemented by the US FDA in 1995 as the "Threshold of Regulation" for food contact materials (US FDA, 1995) based on a distribution plot of the chronic dose rates from the analysis of 343 carcinogens from the carcinogenic potency database derived by Gold et al (1984) and extrapolated to a distribution of $1 \times 10^{-6}$ risk. In Canada, food packaging material constituents (i.e. indirect food contact materials) that have dietary exposures falling below $0.025 \mu \mathrm{g} / \mathrm{kg}$ bw/day (equivalent to $1.5 \mu \mathrm{g} /$ person/day) do not require the submission of toxicological data to establish safety. In pre-market cases where evidence suggests that a component or impurity of a packaging material is a non-threshold carcinogen, risk assessments are conducted to evaluate whether the lifetime cancer risk is significantly below one in a million (e.g. Lifetime cancer risk $<10^{6}$ for azo-type dyes in polystyrene packaging) or letters of no objection are not issued. In the post-market context, the TTC approach is suggested to be useful when biological data are limited but the chemical structure is known and there are sufficient exposure data as a supplement to prioritisation of risk management actions (for example in association with ALARA) (O’Brien et al., 2006).

Another approach identified in scientific literature for risk characterisation of non-threshold compounds which lack carcinogenicity studies is based on the correlation between in vivo genotoxic potency with carcinogenic potency (Sanner and Dybing, 2005). This approach has not been used by BCS. This approach is based on information from the IARC Monographs on the evaluation of carcinogenic risks to humans (http://monographs.iarc.fr) which include descriptions of genotoxicity experiments together with the lowest effective dose (LED) giving such responses. Twenty-eight orally administered carcinogens evaluated by IARC were identified to have reported LED values from in vivo genotoxicity studies (Sanner and Dybing, 2005). When these 28 LED values were plotted versus their respective T25 values in a logarithmic plot, the linear regression showed a good correlation between the two sets of values $\left(\mathrm{r}^{2}=0.71\right)$. In such cases, it is suggested that the LED divided by a specified assessment factor may represent a virtually safe level or a tolerable risk level for a possible carcinogenic effect (Dybing et al., 2008). In a critique of this approach by the U.K. Committee on Mutagenicity of Chemicals in Food, Consumer Products and the Environment (COM), it was noted that there is no proposed assessment factor published in the paper by Sanner and Dybing for mutagens and the selection of the chemicals used for the comparison of LED and T25 isn't fully explained (COM, 2006). Continued work in this area is required in order for this approach to be a useful risk characterization tool.

The application of the margin of exposure (MOE) approach has gained international support, to address the disadvantages of the ALARA principle (discussed in the 
previous section) and the numerical estimation of risk to humans. The MOE is the ratio between a dose leading to a specified tumours incidence in experimental animals and human exposure. The MOE is considered the most scientifically credible approach to the formulation of advice to risk managers because it takes into account intake/exposure and the available data on the doseresponse relationship without extrapolation or the generation of possibly uncertain risk estimates. The EWI conference considered that a BMDL is the most appropriate reference point for calculating a MOE. The T25 could also be used but it was noted that the resulting value would require a different interpretation from a MOE derived from a BMDL10 as it is based on a different level of response and is usually derived from fewer data points.

Because the MOE is a ratio, it is a dimensionless number and there was a consensus at the EWI conference that the MOE should be accompanied by a narrative to aid interpretation. For instance, the narrative should include a discussion of inherent uncertainties when the MOE is based on animal data (e.g. species differences and human variability). In addition, after taking into account uncertainties related to the precision of the dose-response relationship and the quality of the human exposure data, which in some cases may be quite poor, risk managers should be informed of the magnitude of a MOE that could be considered to represent a low priority for risk management actions.

There were differing views among the conference participants about how to interpret the magnitude of a MOE. It was recognised that in general terms the higher the MOE, the lower the degree of concern. It was also noted that MOEs based on data from lifetime animal studies are potentially more conservative if actual human exposures are short in duration or sporadic, rather than lifetime. A number of participants at the EWI conference had reservations about the rationale for the proposal made by the EFSA Scientific Committee in its opinion (EFSA, 2005) that in general a MOE of 10000 or higher, if based on a BMDL10 from an animal study, would be of low concern from a public health point of view and might reasonably be considered as a low priority for risk management actions. JECFA has previously, at its $64^{\text {th }}$ meeting (JECFA, 2005) considered MOEs of 10000 or higher for unintended contaminants (polycyclic aromatic hydrocarbons and ethyl carbamate from food, excluding alcoholic beverages) to be of low concern for human health. The justification for attributing low concern to MOEs at or above 10000 is not fully supported scientifically. The EWI conference questioned whether the EFSA opinion was more a practical suggestion of where the division between lesser concern and greater concern might lie.
The EWI conference emphasized that the figure of 10000 should not be viewed as a threshold for triggering concern or risk management action. For example, a high MOE should not preclude consideration of risk management action, including the application of ALARA (discussed above). The MOE approach is intended to provide consistent methodology for assessing the risk posed by genotoxic carcinogens and prioritizing compounds for risk management action.

BCS has derived MOEs for the risk characterization of food contaminants that are considered non-threshold carcinogens. Interpretation of the risk characterized by the MOE is on a case-by-case basis dependent on the consideration given to uncertainties within the database and exposure estimations. As expressed at the EWI conference, further guidance for the interpretation of the magnitude of the MOE in relation to the degree of concern from a public health point of view would be beneficial.

Within Health Canada a prioritization scheme for the risk management of non-threshold carcinogens in a postmarket context has been established under CEPA and is available on the internet at : http://www.hc-sc.gc.ca/ewhsemt/pubs/contaminants/approach/index-eng.php. In this approach the carcinogenic and mutagenic potency of compounds are compared to the estimated daily intake by the general population (referred to as the Exposure/Potency Index or EPI). Potency is expressed as the concentration or dose which induces a $5 \%$ increase in the incidence of, or deaths due to, tumours or heritable mutations considered to be associated with exposure. The TD05 is not based on the confidence limit but, rather, is computed directly from the curve. This was considered to be appropriate in view of the stability of the data in the experimental range and to avoid unnecessarily conservative assumptions. The value of $5 \%$ is arbitrary. The priority for further action (i.e. analysis of options to reduce exposure) is considered to be high for EPIs of approximately $2.0 \times 10^{-4}$ or greater; for EPIs within the range of greater than or equal to approximately $2.0 \times 10^{-6}$ to less than approximately $2.0 \times 10^{-4}$, it is considered to be moderate and for EPIs less than approximately $2.0 \times 10^{-6}$ it is considered to be low. Obviating the establishment of a single deminimis risk level enables the assessment of nonthreshold toxicants to be based to the extent possible on scientific considerations. Similar to the issues raised regarding a lack of scientific basis for an MOE of 10000 to indicate low concern, the scientific justification is not readily available for the levels used in the CEPA prioritization scheme. However, the CEPA approach provides a more structured and consistent tool for prioritization.

The MOE or EPI of a food contaminant should not be the basis for approving the deliberate addition or use of 
genotoxic carcinogens in food, for condoning infringements of regulations or for relaxing standards.

\section{Risk Management}

Recommendations for risk reduction are made when exposure poses a possible health risk, as identified and characterized in the risk assessment. Risk management priorities for risk reduction actions depend on the extent and frequency that the TDI or upper bound estimate is exceeded or on the magnitude of the MOE (Health Canada, 1994). There are a variety of risk management options that help to ensure a safe food supply. These range from preventative (setting regulatory limits, developing codes of practice for producers or manufacturers, issuing consumer advisories) to restorative (product recall or reformulation). Risk management goals are established once the situation has been identified in an appropriate context. Consideration should be given to the needs, issues, and concerns of interested and affected parties, the nature of the decisions that have to be made, and any assumptions and constraints governing the decision (Health Canada, 2000).

The Food Directorate continues to maintain the position that levels of food contaminants should be minimized as much as possible and uses this principle to guide risk management options. For example, when benzene levels were reported in beverages, industry took action to reformulate the products and freeze the shipment of contaminated product to retail outlets. The Food Directorate developed more sensitive benzene detection methods and conducted a follow-up survey to ensure these products would be of negligible concern to human health. Furthermore, the Food Directorate and the Canadian Food Inspection Agency (CFIA) continue to work with the beverage industry to ensure that the formation of benzene during manufacturing is minimized.

Regardless of the situation, the primary goal of any risk management strategy is to ensure an appropriate level of health protection (Health Canada, 2000).

\section{Discussion \& Conclusion}

This document presented a general overview of international approaches for the post-market risk assessment of genotoxic carcinogenic contaminants in food, consistent with EFSA, WHO and ILSI Europe, with a focus on the four components of a risk assessment: hazard identification, hazard characterization (doseresponse assessment), exposure assessment, and risk characterization. This report was written to contribute to Health Canada's department-wide discussions on the standardization of the decision-making process for the risk management of genotoxic carcinogens and to provide the scientific background for a departmental report on Food Directorate policy.

Because technological advances have made it possible to detect extremely low levels of contaminants in food, risk perception and tolerance must also evolve because zero risk is not a realistic target for the post-market management of genotoxic carcinogens. Current risk assessment and management practices for genotoxic carcinogens are protectively conservative but don't effectively incorporate the concept of prioritizing higher risk scenarios for management action.

As discussed, considerable international work has been conducted in this field. Although there is at present no consensus with regard to the scientific justification for determining whether a risk is low, medium or high, work in this area is ongoing.

There are several areas in the risk assessment paradigm where the development of department-wide guidance would be beneficial to current Food Directorate practices. These areas include refining how weight-of-evidence is used to determine whether a compound should be considered a non-threshold carcinogen, elaborating on the technical criteria for choosing the appropriate doseresponse assessment approach, and establishing a consistent approach for interpreting and prioritizing risk.

\section{Acknowledgements}

The authors wish to thank Joel Rotstein, Madeline Weld and Mark Feeley (Bureau of Chemical Safety), Joan Wong and Catherine Adcock (Pest Management Regulatory Agency) and Diane Bedford (European Food Safety Authority) for their many helpful contributions to this paper.

\section{References}

[1] Abt, E., Rodricks, J.V., Levy, J.I., Zeise, L. and Burke, T.A. (2010) Science and decisions: advancing risk assessment. Risk Analysis Jul: 30(7): 1028-36.

[2] Barlow, S., Dybing, E., Edler, L., Eisenbrand, G., Kroes, R. and van den Brandt, P. (2002a) Food safety in Europe (FOSIE): risk assessment of chemical in food and diet. Food and Chemical Toxicology 2/3, 141-427.

[3] Barlow, S.M., Grieg, J.B., Bridges, J.W., Carere, A., Carpy, A.J.M., Galli, C.L., Kleiner, J., Knudsen, I., Koeter, H.B.W.M., Levy, L.S., Madsen, C., Mayer, S., Narbonne, J.,-F., Pfannbuch, F., Prodanchuk, M.G., Smith, M.R. and Steinberg, P. (2002b) Hazard identification by methods of animal-based toxicology. Food and Chemical Toxicology 40 (2/3), 145-191. 
[4] Barlow, S., Renwick, A.G., Kleiner, J., Bridges, J.W., Busk, L., Dybing, E., Edler, L., Eisenbrand, G., FinkGremmels, J., Knaap, A., Kroes, R., Liem, D., Muller, D.J.G., Page, S., Rolland, V., Schlatter, J., Tritscher, A., Tueting, W. And Wurtzen, G. (2006) Risk assessment of substances that are both genotoxic and carcinogenic Report of an International Conference organized by EFSA and WHO with support of ILSI Europe. Food and Chemical Toxicology 44, 1636-1650.

[5] Bolt, H.M. and Degen, G. H. (2004) Human carcinogenic risk evaluation, part II: contributions of the EUROTOX specialty section for carcinogenesis. Toxicol. Sci., 81, 3-6.

[6] Boobis, A.R., Cohen, S.M., Dellarco, V., McGregor, D., Meek, M.E., Vickers, C., Willcocks, D. and Farland, W. (2006) IPCS framework for analyzing the relevance of a cancer mode of action for humans. Crit. Rev. Tox. 36, 781-792.

[7] Bos, P.M.J., Baars, B., Marcel, T.M. and van Raaij, M.T.M. (2004) Risk Assessment of Peak Exposure to Genotoxic Carcinogens: A Pragmatic Approach. Toxicol Letters 151:43-50.

[8] Brambilla, G. and Martelli, A. (2004) Failure of the standard battery of short-term tests in detecting some rodent and human genotoxic carcinogens. Toxicology 196, 1-19.

[9] Brusick, D. (2001) Genetic toxicology. In: Wallace Hayes, A. (Ed.), Principles and Methods of Toxicology, fourth ed. Taylor \& Francis, Philadelphia, pp. 819-852.

[10] CEPA (1994) Canadian Environmental protection Act, Health Canada, Human health risk assessment for priority substances, Minister of Supply and Services Canada, Canadian Communication Group, Ottawa, Ontario. Cat. No En40-215/41E.36. (http://www.hc-sc.gc.ca/ewhsemt/pubs/contaminants/approach/index-eng.php).

[11] COM, Committee on mutagenicity of chemicals in food consumer products and the environment. (2006). The lowest effective dose (LED for in vivo genotoxicity); a possible approach to mutagen potency ranking. (http://www.advisorybodies.doh.gov.uk/pdfs/mut070 2.pdf)

[12] Dearfield, K.L., Auletta, A.E., Cimino, M.C. and Moore, M.M. (1991) Considerations in the U.S. Environmental Protection Agency's testing approach for mutagenicity. Mutation Research 258, 259-283.

[13] Douglass, J.S. and Tennant, D.R. (1997) Estimation of dietary intake of food chemicals. In: Tennant, D.R. (Ed.), Food Chemical Risk Analysis, Blackie Academic and Professional. Chapman and Hall, London, pp. 195-216.

[14] Dybing, E., Doe, J., Groten, J., Kleiner, J., O'Brein, J., Renwick, A.G., Schatter, J., Steinberg, P., Tritscher, A., Walker, R., Younes, M. (2002) Hazard characterization of chemicals in food and diet: dose response, mechanisms and extrapolation issues. Food and Chemical Toxicology 40, 237-282.

[15] Dybing, E., O'Brien, J., Renwick, A.G., and Sanner, T. (2008) Risk assessment of dietary exposures to compounds that are genotoxic and carcinogenic - An overview, Toxicology Letters, 180, 110-117.

[16] Edler, L., Poirier, K., Dourson, M., Kleiner, J., Mileson, B., Nordmann, H., Renwick, A., Slob, W., Walton, K. And Wurtzen, G. (2002) Mathematical modelling and quantitative methods, Food Chem. Toxicol., 40, 283-326.

[17] EFSA (2005) European Food Safety Authority, Opinion of the Scientific Committee on a request from EFSA related to a harmonised approach for risk assessment of substances which are both genotoxic and carcinogenic. Request No EFSA-Q-2004-020. $\begin{array}{llll}\text { Adopted on } & 18 & \text { October } & 2005 .\end{array}$ (http://www.efsa.eu.int/science/sc_commitee/ sc_opinions/1201_en.html)

[18] EHC 240 (2009) Environmental Health Criteria; 240, Principles and methods for the risk assessment of chemicals in food. ISNB 978924157240 8. World Health Organization 2009.

[19] (http://whqlibdoc.who.int/ehc/WHO_EHC_240_6_e ng_Chapter3.pdf)

[20] EPA (2005a) US Environmental Protection Agency. Guidelines for Carcinogen Risk Assessment, Risk Assessment Forum, Washington, DC.

[21] (http://www.epa.gov/cancerguidelines/).

[22] EPA (2005b) US Environmental protection Agency. Supplemental Guidance for Assessing Susceptibility from early-Life exposure to carcinogens. Risk Assessment Forum. Washington, DC. (http://www.epa.gov/raf/publications/pdfs/childrens_ supplement_final.pdf)

[23] EC (European Commission). Scientific Committee on Consumer Products (SCCP), Scientific Committee on Health and Evironmental Risks (SCHER) and Scientific Committee on Emerging and Newly Identified Health Risks (SCENIHR) (SCCP). (2008). Draft opinion on use of the threshold of toxicological concern (TTC) approach for the safety assessment of chemical substances. (http://ec.europa.eu/health/ph_risk/committees/docu ments/sc_o_001.pdf).

[24] Gold, L.S., Sawyer, C.B., Magaw, R., Backman, G.M., de Veciana, M., Levinson, R., Hooper, N.K., Havender, W.R., Bernstein, L., Peto, R., Pike, M., and Ames, B.N. (1984). A carcinogenesis potency database of the standardized results of animal bioassays. Environmental Health Perspectives 58, 9-319.

[25] Health Canada (2000) Health Canada Decisionmaking framework for identifying, assessing and managing health risk, August 1, 2000. (http://www.hssc.gc.ca/aha-asc/pubs/hpfb-dgpsa/riskrisques_3_e.html) 
[26] Hengstler, J.G., Bogdanffy M.S., Bolt, H.M. and Oesch, F. (2003) Challenging dogma: thresholds for genotoxic carcinogens? The case of vinyl acetate. Annu. Rev. Pharmacol. Toxicol., 43, 485-520.

[27] IARC (2008) IARC Monographs on the Evaluation of Carcinogenic Risks to Humans, Vol 97. 1,3Butadiene, Ethylene Oxide and Vinyl Halides (Vinyl Fluoride, Vinyl Chloride and Vinyl Bromide), Lyon, International Agency for Research on Cancer, 5-12 June 2007.

[28] JECFA (2005). Joint FAO/WHO Expert Committee on Food Additives, Sixty-fourth meeting, Rome, 8-17 February 2005.

(http://www.who.int/ipcs/food/jecfa/summaries/sum mary_report_64_final.pdf)

[29] Kirsch-Volders,M., Sofuni,T., Aardema,M., Albertini,S., Eastmond,D., Fenech, M., Ishidate,M.Jr, Lorge,E., Norppa,H., Surallés,J., von der Hude,W. and Wakata,A. (2000) Report from the in vitro micronucleus assay working group. Mutat. Res., 35, 167-172.

[30] Kroes, R., Muller, L., Lambe, J., Lowik, M.R.H., van Klaveren, J., Kleiner, J., massey, R., Mayer, S., Urieta, I., Verger, P. and Visconti, A. (2002) Assessment of intake from the diet. Food and Chemical Toxicology $40(2 / 3), 327-385$.

[31] Kuiper-Goodman, T. (2004) Risk assessment and risk manaement of mycotoxins in food. In: Magan N. And Olsen M. (Ed.) Mycotoxins in food: detection and control. WoodHead publishing Limited, Cambrige, England, pp 3-31.

[32] Kuiper-Goodman, T., Hilts, C., Billiard, S.M., Kiparissis, Y., Richard, I. D. K., and Hatward, S. (2010). Health risk assessment of ochratoxin A for all age-sex strata in a market economy, Food Adiitives and Contaminants: Part A, 27: 2, 212-240.

[33] Li-Muller, A., Healy, N., Yole, M. and Petrovic, S. (2011). Draft CSD Interim Position Paper on Human Health Risk Assessment for Short-term Exposure to Carcinogens at Contaminated Sites.

[34] Lobenhofer, E.K., Cui, X., Bennett, L., Cable, P.L., Merrick, B.A., Churchill, G.A., Afshari, C.A. (2004) Exploration of low-dose estrogen effects:identification of No Observable Transcriptional Effect Level (NOTEL). Toxicol. Pathol. 32, 482-492.

[35] Meek, M.E., Bucher, J.R., Cohen, S.M., Dellarco, V., Hill, R.N., Lehman-Mckeeman, L.D., Longfellow, D.G., Pastoor, T., Seed, J. And Patton, D.E. (2003) A framework for human relevance analysis of information on carcinogenic modes of action, Crit. Rev. Toxicol., 33, 591-653.

[36] Meek, M. E. B. (2008) Recent Developments in Frameworks to Consider Human Relevance of Hypothesized Modes of Action for Tumours in Animals, Enviro. Mol. Mut. 49, 110-116.
[37] NRC (1983) U.S. National Research Council. Risk Assessment in the Federal Government: Managing the Process, (also known as "The Red Book"), National Academy Press, Washington, DC.

[38] NRC (2009) U.S. National Research Council. Science and Decisions:Advancing Risk Assessment. National Academy Press, Washington, DC.

[39] O’Brien, J., Renwick, A.G., Constable, A., Dybing, E., Muller, D.J.G., Schlatter, J., Slob, W., Tueting, W., van Benthem, J., Williams, G.M. and Wolfreys, A. (2006) Approaches to the risk assessment of genotoxic carcinogens in food: A critical appraisal. Food and Chemical Toxicology 44, 1613-1635.

[40] OECD (2002) Guidance notes for analysis and evaluation of chronic toxicity and carcinogenicity studies, OECD Environment, Health and Safety Publications. Series on Testing and assessment No. 35. (http://www.olis.oecd.org/olis/2002doc.nsf/LinkTo/en v-jm-mono(2002)19.)

[41] OECD (2009) OECD draft guidance document $\mathrm{N}^{\circ}$ 116 on the design and conduct of chronic toxicity and carcinogenicity studies, supporting TG 451, 452 and 453. (http://www.oecd.org/dataoecd/57/32/44076587.pdf)

[42] Petersen, B. (2002) Estimating dietary exposure: methods, algorithms and general considerations. In: Paustenback, D. (Ed.), Human and Ecological Risk Assessment: Theory and Practice. Wiley Interscience, New York, pp. 895-912.

[43] Renwick, A.G., Barlow, S., Hertz-Picciotto, I., Boobis, A.R., Dybing, E., Edler, L., Eisenbrand, G., Grieg, J.B., Kleiner, J., Lambe, J., Muller, D.J.G., Smith, M.R., Tritscher, A., Tuijelaars, S., van den Brandt, P.A., Walker, R. and Kroes, R. (2003) Risk characteristion of chemicals in food and diet. Food and Chemical Toxicology 41, 1211-1271.

[44] Rhomberg, L.R., Baetcke, K., Blancato, J., Bus, J., Cohen, S., Conolly, R., Dixit, R., Doe, J., Ekelman, K., Fenner-Crisp, P., Harvey, P., Hattis, D., Jacobs, A. and Jacobson-Kram, D. (2007) Issues in the design and interpretation of chronic toxicity and carcinogenicity studies in rodents: approaches to dose selection. Crit. Rev. Tox. 37, 729-837.

[45] Sander, M., Cadet, J., Casciano, D.A., Galloway, S.M., Marnett, L.J., Novak, R.F., Pettit, S.D., Preston, R.J., Skare, J., Williams, G.M., Van Houten, B., Gollapudi, B.B. (2005) Proceedings of a workshop on DNA adducts:biological significance and applications to risk assessment, Washington, DC, April 13-14, 2004. Toxicol. Appl. Pharmacol. 208, 1-20.

[46] Sanner, T., and Dybing, E. (2005) Comparison of carcinogenic and in vivo genotoxic potency estimates. Basic Clin. Pharmacol. Toxicol. 96, 131-139.

[47] Schlosser, P.M. and Bogdanffy, M.S. (1999) Determining modes of action for biologically based risk assessments, Regul. Toxicol. Pharmacol., 30, 75-79. 
[48] Seed, J., Carney, E.W., Crofton, K.M., DeSesso, J.M., Foster, P.M.D., Kavlock, R., Kimmel, G., Klaunig, G., Meek, M.E., Preston, R.J., Slikker, W., Tabacova, S. and Williams, G.M. (2005) Overview:using mode of action and life stage information to evaluate the human relevance of animal toxicity data. Crit. Rev. Tox. 35, 663-672.

[49] Seiler, J.P. (1977) Inhibition of testicular DNA synthesis by chemical mutagens and carcinogens. Preliminary results in the validation of a novel shortterm test. Mutat. Res., 46, 305-310.

[50] Smith, B., Cadby, P., Leblanc, J-C., and Setzer, R.W. (2010). Application of the margin of exposure (MOE) approach to substances in food that are genotoxic and carcinogenic, example: methyleugenol, CASRN:93-152, Food and Chemical Toxicology, 48, S89-S97.

[51] Sonich-Mullin, C., Fielder R., Wiste, J., Baetcke, K., Dempsey, J., Fenner-Crisp, P., Grant, D., Hartley, M., Knaap, A., Kroese, D., mangelsdorf, I., Meek, E., Rice, J.M. and Younes, M. (2001) IPCS conceptual framework for evaluating a MOA for chemical carcinogenesis, Regul. Toxicol. Pharmacol., 34, 146152.

[52] US FDA (1995) Food Additives: Threshold of regulation of substances used in food contactarticles: Final rule. Frederal register. 60:36582-36596. (www.cfsan.fda.gov/ Ird/t36582.html)

[53] US FDA (2000) Toxicological Principles for the safety Assessment of Food Ingredients - Redbook 2000. IV.C.6 Carcinogenicity Studies with Rodents. (http://vm.cfsan.fda.gov/ redbook/red-toca.html)

[54] WHO (1987) Principles for the safety assessment of food additives and contaminants in food, Environmental Health Criteria, 70, Geneva, World Health Organization.
[55] WHO (1995) World Health Organization, Report of a workshop in the frame of GEMS/Food-Euro, Kulmbach, Germany, 26-27 May 1995. (http://www.who.int/foodsafety/publications/chem/lo wlevel_may1995/en/index.html)

[56] WHO (1999) Principles for the assessment of risks to human health from exposure to chemicals, Enviornmental Health Criteria, 210, Geneva, World Health Organization (http://www.inchem.org/documents/ech/ech210.htm)

[57] WHO (2005) World Health Organization, Guidance document for the use of data in development of chemical-specific adjustment factors (CSAFs) for interspecies differences and human variability in dose/concentration-response assessments. (www.inchem.org/documents/harmproj/harmproj/har mproj2.pdf)

[58] WHO and FAO (2009) World Health Organization and Food and Agriculture Organization of the United Nations; Environmental Health Criteria; 240, Principles and methods for the risk assessment of chemicals in food. ISNB 9789241572408.

[59] (http://whqlibdoc.who.int/ehc/WHO_EHC_240_6_e ng_Chapter3.pdf)

[60] Williams, G.M. (1999) Mechanistic considerations in cancer risk assessment. Inhal. Toxicol. 11, 549-554.

[61] Williams, G.M. (2001) Mechanisms of chemical carcinogenesis and application to human cancer risk assessment. Toxicology 166, 3-10.

[62] Williams, G.M., Iatropoulos, M.J., Jeffery, A.M. (2005) Thresholds for DNA-reactive (genotoxic) organic carcinogens. J. Toxicol. Pathol. 18, 69-77.

[63] Williams, G.M. (2008) Application of mode of action considerations in human cancer risk assessment. Toxicology Letters, 180, 75-80. 